\title{
Induced Dynamic Nonlinear Ground Response at Garner Valley, California
}

\author{
by Zack Lawrence, Paul Bodin, Charles A. Langston, Fred Pearce, Joan Gomberg, \\ Paul A. Johnson, Farn-Yuh Menq, and Thomas Brackman
}

\begin{abstract}
We present results from a prototype experiment in which we actively induce, observe, and quantify in situ nonlinear sediment response in the near surface. This experiment was part of a suite of experiments conducted during August 2004 in Garner Valley, California, using a large mobile shaker truck from the Network for Earthquake Engineering Simulation (NEES) facility. We deployed a dense accelerometer array within meters of the mobile shaker truck to replicate a controlled, laboratory-style soil dynamics experiment in order to observe wave-amplitudedependent sediment properties. Ground motion exceeding $1 g$ acceleration was produced near the shaker truck. The wave field was dominated by Rayleigh surface waves and ground motions were strong enough to produce observable nonlinear changes in wave velocity. We found that as the force load of the shaker increased, the Rayleighwave phase velocity decreased by as much as $\sim 30 \%$ at the highest frequencies used (up to $30 \mathrm{~Hz}$ ). Phase velocity dispersion curves were inverted for $S$-wave velocity as a function of depth using a simple isotropic elastic model to estimate the depth dependence of changes to the velocity structure. The greatest change in velocity occurred nearest the surface, within the upper $4 \mathrm{~m}$. These estimated $S$-wave velocity values were used with estimates of surface strain to compare with laboratory-based shear modulus reduction measurements from the same site. Our results suggest that it may be possible to characterize nonlinear soil properties in situ using a noninvasive field technique.
\end{abstract}

\section{Introduction}

Nonlinear response resulting from large strain deformations in samples taken from near-surface sedimentary deposits has been observed in laboratory experiments for many years (Seed and Idriss, 1970; Hardin and Drnevich, 1972a, b; Vucetic, 1994; Guyer and Johnson, 1999; Ostrovsky and Johnson, 2001). The complicated nature of nonlinearity is typically characterized by simple curves that relate a change in elastic modulus and damping to the applied strain level (e.g., Beresnev and Wen, 1996). Seismic hazard assessments use these curves to understand how a site will behave during strong earthquake ground motions, typically reducing the peak ground motions one would expect if using a linear response model (Kramer, 1996).

It is not fully understood how closely the laboratory measurements reflect in situ nonlinear behavior (Beresnev and Wen, 1996; Field et al., 1998). In situ observations of nonlinear ground response during large earthquakes, once rare, have recently increased in number due to advances in deploying strong-motion networks (Aguirre and Irikura, 1997; Field et al., 1997; Frankel et al., 2002; Rubinstein and Beroza, 2004; Bonilla et al., 2005). The lack of experimental control inherently limits the use of large earthquakes in nonlinear response studies. Earthquakes are unpredictable and occur infrequently, particularly near a suitable strongmotion network. The earthquake source and wave propagation involved produces a complex signal that is difficult to analyze, is irregular in time, and contains a variety of interacting waves. Such complexities have made it difficult to unambiguously observe in situ nonlinear response from earthquake sources.

A different approach, and one we take in this study, has the potential to allow researchers an alternative method to observe and investigate in situ nonlinear seismic wave propagation. The unique dataset collected could provide further insight into how closely laboratory measurements reflect in situ nonlinear behavior. In our approach, we generate strong ground motions capable of producing nonlinear effects using a large mobile shaker truck that is commonly employed in active seismic imaging. The shaker truck produces a controlled dynamic load with uniform amplitude 
over a range of frequencies and is easily driven to a test site. Dimitriu (1990) was the first to implement this field approach with mixed results. More recently, the University of Texas at Austin and the Network for Earthquake Engineering Simulation (NEES) have advanced the ability to study in situ nonlinear ground response and liquefaction potential with the availability of state-of-the-art dynamic mobile shaker trucks (Stokoe, Rathje, et al., 2004).

This article describes our proof-of-concept study conducted at Garner Valley, California, in August 2004. The study was part of a demonstration project, consisting of five multidisciplinary experiments, showcasing the use of NEES shaker trucks in earthquake science and engineering (Gomberg et al., 2004). The primary focus of this prototype study is threefold. First, we test the ability of the NEES mobile shaker truck, called T-Rex, to induce in situ nonlinear response in the near-surface sediments. Second, we test the ability of a surface-mounted strong-motion array to record the strong ground motions produced by T-Rex. Third, we investigate how to quantify the nonlinear response using the observed wave-field characteristics.

The in situ nonlinear response in the shallow sediments $(<4 \mathrm{~m})$ is quantified by changes in the wave propagation velocity of high-strain seismic waves at different applied force loads. We find that the waves slow down as the applied force load is increased and that sediment properties are waveamplitude dependent. The near-source wave field is found to be dominated by Rayleigh surface waves. Thus, we calculate the Rayleigh phase velocity spectrum at different applied force loads and invert the dispersion curves to model changes to the shallow shear $(S)$ wave velocity structure due to the induced nonlinear response. We conclude with an estimate of in situ shear modulus reduction as a function of strain level, and we explore if and how they compare to measurements made with laboratory methods on sediments from the same site.

\section{Study Area}

Our field test site was located at Garner Valley, California, which is an intermountain valley located within the Peninsular Ranges Batholith in southern California. Garner Valley contains an ancestral lake bed with soft alluvium in the upper $\sim 20 \mathrm{~m}$ overlaying weathered and competent granite (Steidl et al., 1996). Soil types in the top $\sim 20 \mathrm{~m}$ include silty sand, sand, clayey sand, and silty gravel (J. Steidl, personal comm., 2004).

\section{Experimental Equipment and Configuration}

Figure 1 is a field photo taken during data collection. It shows the key experimental equipment used in our study. T-Rex is a triaxial mobile shaker truck capable of producing harmonic vibrations in three directions (two horizontal and one vertical) at variable ground forces ranging up to

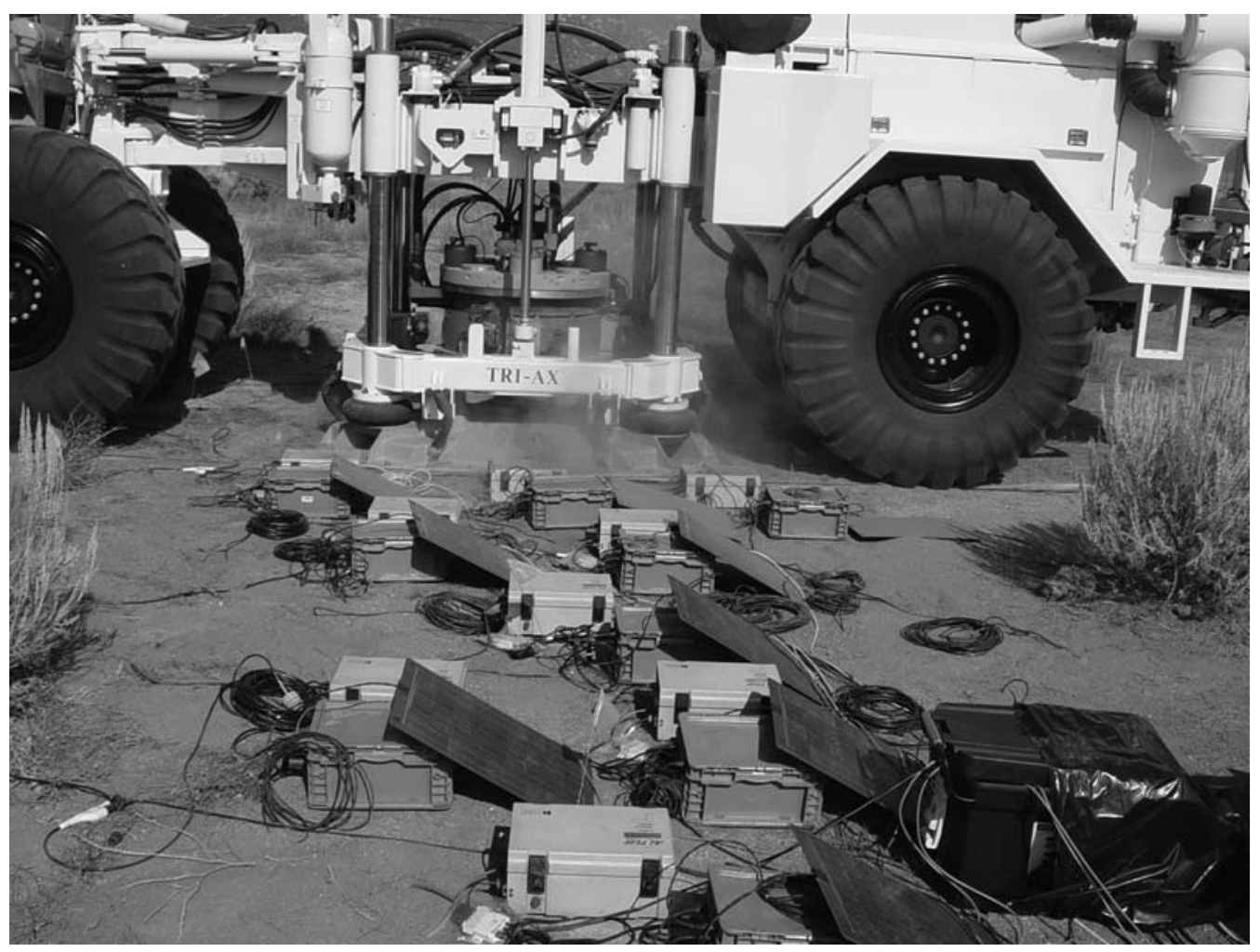

Figure 1. Photo of the experiment. Shown is the NEES mobile shaker truck, T-Rex. Adjacent to T-Rex is the accelerometer array installed to record the induced high-strain seismic waves. Each three-component K2 accelerometer has an external battery and solar panel attached. 
$60,000 \mathrm{lb}$ vertically and 30,000 lb horizontally. Nominally, T-Rex produces a constant force output for frequencies between 5 and $180 \mathrm{~Hz}$ for horizontal motions, between 12 and $180 \mathrm{~Hz}$ for vertical motions, and the force response falls off quickly outside of those ranges (Fig. 2). In practice, the force response is commonly observed to be site specific and to be a function of the stiffness of the soil beneath the baseplate. Calculated values of applied ground force suggest that the theoretical force response curve is reasonable for our test site.

The strong ground motions created near T-Rex were recorded on an adjacent surface array of nine Kinemetrics K2 accelerometers. Figure $3 \mathrm{a}$ is a schematic of the accelerometer array showing the station identification numbers used in the field, in the archived data, and referred to later in this article. Each accelerometer recorded three components of motion at 200 samples per second with a full scale of $\pm 2 g$ of ground acceleration. The array spanned the width of the baseplate $(2.4 \mathrm{~m})$ and extended $4 \mathrm{~m}$ away from T-Rex in the radial direction. Three of the accelerometers were installed as close to T-Rex as possible without risking harm to the instruments $(\sim 0.2 \mathrm{~m}$ and $\sim 0.5 \mathrm{~m}$ to the edge and center of each accelerometer, respectively). Each accelerometer in the array was firmly bolted to a concrete pad $(\sim 0.25 \mathrm{~m}$ in diameter and $\sim 0.05$-m deep) that was secured to the ground with three 0.5-m-long metal tubes (Fig. 3b). To decrease the possible impedance contrast between the concrete instrument pad and the surrounding soil, low-density lava rock was used in the concrete mix. The concrete was allowed to cure fully for a month before the experiment. The array design and construction was intended to increase the chance of observing nonlinear ground response by withstanding and recording on scale the largest ground motions T-Rex could produce. We feel we were successful in that regard because after

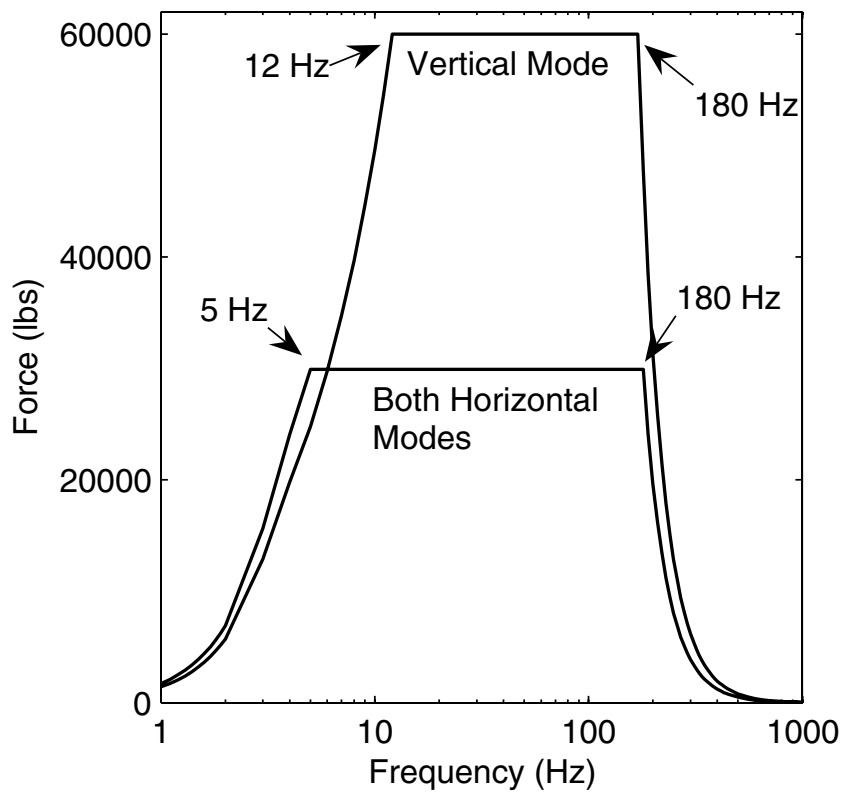

Figure 2. T-Rex force output in units of pounds as a function of frequency (Stokoe, Rathje, et al., 2004).

\section{(a) Array Layout and Station Identification Numbers}

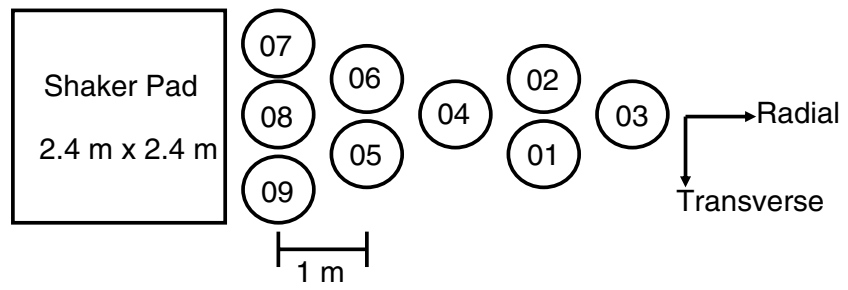

(b) Instrument Pad Cross-Section

(not to scale, 2:1 horizontal exaggeration)

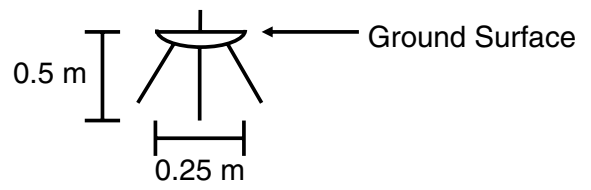

Figure 3. (a) Schematic diagram showing the layout and station identifications of the surface-mounted accelerometer array located adjacent to T-Rex. Radial and transverse directions of motion are defined. (b) A cartoon cross section of the concrete instrument pad used for each station.

the experiment was concluded, the instrument pads were found to be unharmed and intact with the $\mathrm{K} 2$ accelerometers still bolted firmly in place.

\section{Ground Motions and Wave Propagation}

We will focus on the data from a vertically excited harmonic step-sweep signal at five increasing applied force loads (approximately 12,000, 24,000, 36,000, 48,000, and $60,000 \mathrm{lb}$ ). A step-sweep signal is one where the frequencies are incremented (or, in our case, decremented) in discrete intervals using multiple cycles at each frequency step. The step-sweep signal we used starts at $30 \mathrm{~Hz}$ and downsweeps to $5 \mathrm{~Hz}$ at a step of $0.125 \mathrm{~Hz}$. Approximately 20 cycles are used at each discrete frequency step. This produces a signal $\sim 6.5 \mathrm{~min}$ in duration.

The three-component ground motions recorded at station 08 are shown in Figure 4. Also shown in Figure 4 is the recorded ground force signal produced by T-Rex. The ground force signal is a derived product from NEES accelerometers mounted on the shaker mechanism, the mass of the entire system, and forcing from the hydraulic driving system. The ground force time series represents the force exerted by the pad onto the ground, in this case in units of pounds. The five increasing applied force loads used (approximately $12,000,24,000,36,000,48,000$, and 60,000 lb) are clearly seen in Figure 4 through increasing amplitude of the sweep waveforms on the four traces. Waveforms in Figure 4 are typical for all stations, though note that station 04 experienced data loss during the experiment for the 48,000-lb force load. The details of a single step sweep at $60,000 \mathrm{lb}$ can be more clearly seen in Figure 5. The ground force signal in Figure 5a shows the relative decrease in amplitude as the sweep progresses to frequencies lower than $\sim 12 \mathrm{~Hz}$ as pre- 
(a)

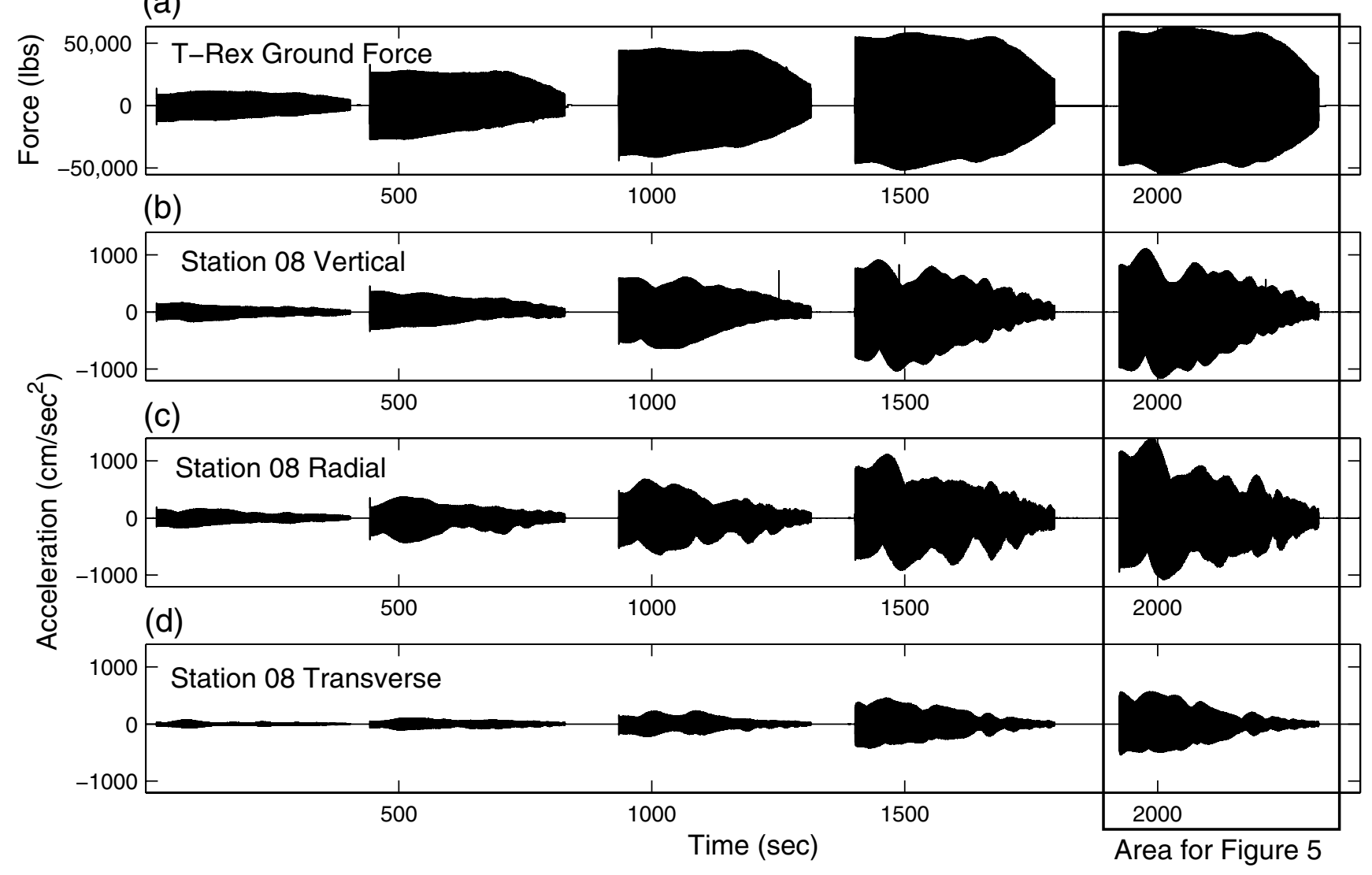

Figure 4. Sample of recorded waveforms. Shown is the $30-5-\mathrm{Hz}$ step-sweep signal at the five applied force loads (at approximately $12,000,24,000,36,000,48,000$, and 60,000 lb). (a) The ground force signal produced by T-Rex. (b) The vertical ground-motion acceleration at station 08. (c) The radial ground-motion acceleration at station 08. (d) The transverse ground-motion acceleration at station 08. Radial and transverse directions are with respect to the propagation of seismic energy away from T-Rex as shown in Figure 3 . Note the large difference in waveform amplitude for the transverse component compared to the vertical and radial components. This is due to Rayleigh waves dominating the wave propagation. The box around the fifth set of waveforms corresponds to Figure 5.

dicted by T-Rex's force response curve (Fig. 2). Vertical and radial ground motions are very similar during the vertical shaking (Fig. 5b and c), while the transverse motion (Fig. 5d) is significantly smaller in amplitude.

T-Rex created ground motions near the truck large enough to induce nonlinear response. During the largest applied force load $(60,000 \mathrm{lb})$, ground accelerations on the vertical and radial components on station 08 exceeded $1.0 \mathrm{~g}$ (Figs. 4 and 5). Ground-motion amplitudes were observed to decrease significantly with distance. At the farthest station in the array, station 03, the largest recorded ground acceleration for any given applied force load was approximately onethird of what was observed closest to T-Rex at stations 07 , 08 , and 09. Nonlinear response has been observed during earthquakes above ground accelerations of $0.1-0.2 g$ (Beresnev and Wen, 1996). The relationship between observing nonlinear soil response and the amplitude of ground accelerations might not be comparable between earthquake induced seismic waves and those created artificially by a shaker truck. The source mechanism and propagating wave field is different and the lower frequency waves produced by an earthquake will sample deeper site structure. However, the fact that we recorded ground accelerations over $1.0 \mathrm{~g}$ and well over the 0.1-0.2g threshold for in situ nonlinearity as stated in Beresnev and Wen (1996) suggests that it is likely that nonlinear response was induced and can be detected in our dataset.

We hypothesize that the wave field created by the vertical shaking is dominated by Rayleigh surface waves. This hypothesis is supported by the observation that the recorded ground motion is largest in the vertical and radial directions (Figs. 4 and 5) and that the particle motion is retrograde elliptical (Fig. 6). Particle motions shown in Figure 6 are for a small time window during $20-\mathrm{Hz}$ shaking for station 08 (closest to T-Rex) and station 03 (furthest from T-Rex). The particle motions are retrograde elliptical and contained nearly entirely in the vertical-radial plane. The lack of transverse displacement is emphasized in the three-dimensional particle motion plots for station 03 .

The particle motion observations reveal some interesting wave effects. The degree of ellipticity and tilt of the particle motion ellipse is not constant throughout the experiment. 

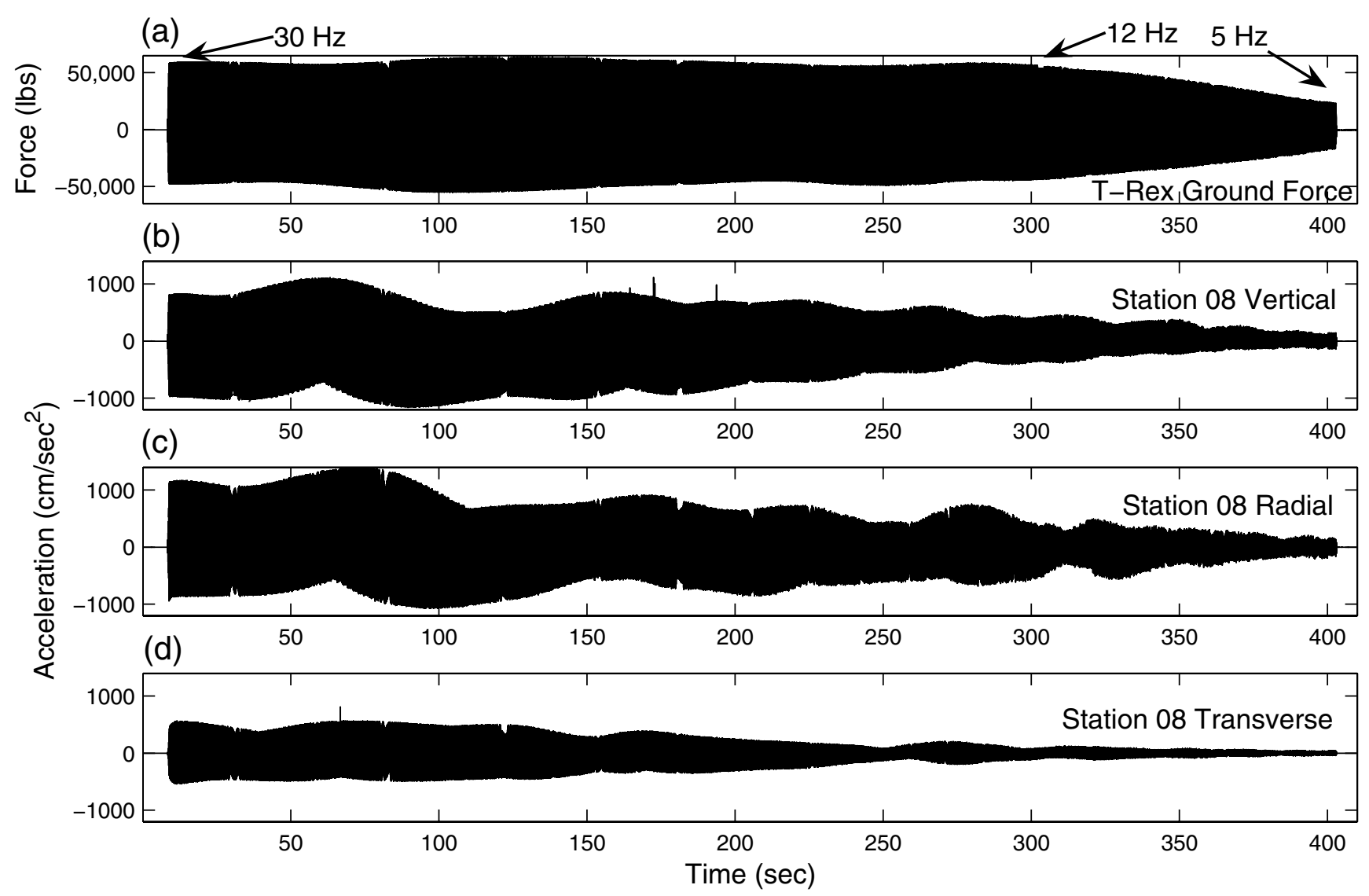

Figure 5. Sample of recorded step-sweep waveforms for the highest applied force load $(60,000 \mathrm{lb})$. Enlarged from the box seen in Figure 4. (a) The ground force signal produced by T-Rex. The step-sweep signal starts at $30 \mathrm{~Hz}$ and downsweeps to $5 \mathrm{~Hz}$. Notice the relative decrease in amplitude below $12 \mathrm{~Hz}$ (see Fig. 2). (b) The vertical ground-motion acceleration at station 08. (c) The radial ground-motion acceleration at station 08. (d) The transverse ground-motion acceleration at station 08. Note the large difference in waveform amplitude for the transverse component compared to the vertical and radial components. This is due to Rayleigh waves dominating the wave propagation.

There is a strong dependence on frequency, distance from TRex, and applied force load. Figure 6 demonstrates the dependence on distance from T-Rex and applied force load. As the applied force is increased, the particle motion ellipses become more elongated and tilted with respect to the ground surface (e.g., Fig. 6b versus Fig. 6h). A similar pattern occurs when looking at stations at a different distance for the same force load (e.g., Fig. 6a versus Fig. 6b). The dynamic characteristic of the observed particle motions, particularly the change in ellipticity and tilt as a function of force load, is an interesting observation. Elastic wave propagation models predict that Rayleigh-wave particle motion ellipses be vertically oriented. The particle motion observations are qualitatively explained by the results from Borcherdt $(1988,2007)$, where he shows that Rayleigh-wave particle motion ellipses become elongated and tilted for high values of attenuation within a viscoelastic half-space. $Q$ values as low as 10 are used to model linear site response in Garner Valley within the shallowest sediments (Steidl et al., 1996). Any induced nonlinearity in the near surface would further lower $Q$ (raise attenuation). This suggests that the particle motions could be used to constrain attenuation and estimate nonlinear damping by using the change in particle motion parameters as a function of strain level. We reserve this analysis for future work.

Our accelerometer array was two dimensional (Figs. 1 and 3). Observationally, a one-dimensional linear array would have sufficed. Stations equidistant from T-Rex have nearly identical observed ground motions. For instance, stations 07,08 , and 09 closest to T-Rex all show nearly identical ground accelerations. Waveforms from all three stations are also in phase with each other. The same holds true for equidistant station pairs 05-06 and 01-02. This is likely due to the baseplate on T-Rex having the same width as the accelerometer array. The wave field propagating away from T-Rex (within the dimensions of our surface array) shows no significant change in phasing or amplitude in the transverse direction.

\section{Phase Velocity Dispersion}

The dominating presence of Rayleigh surface waves in our ground-motion observations naturally leads to the question of whether a change in phase velocity could be used as 
(a)

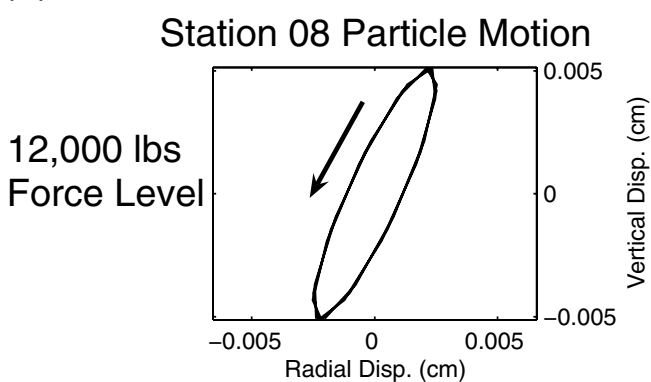

(d)

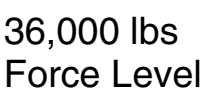

(g)

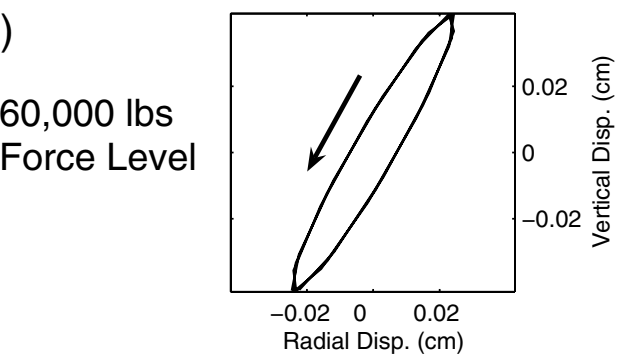

(b)

Station 03 Particle Motion

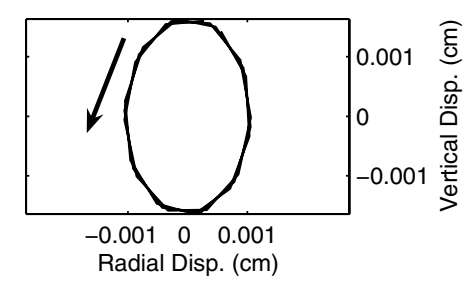

(e)

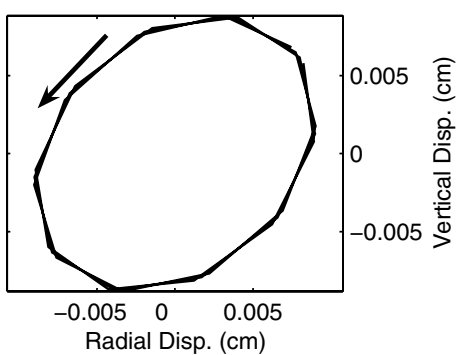

(h)

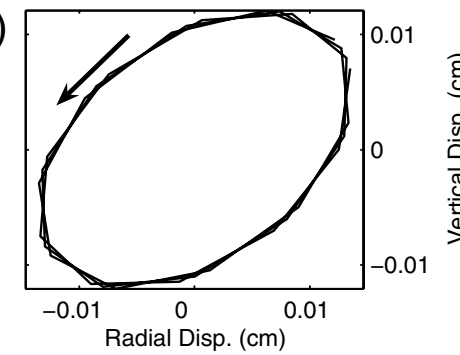

(c) 3-D View of Station 03 Particle Motion

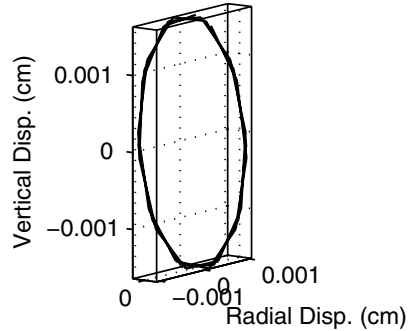

(f)

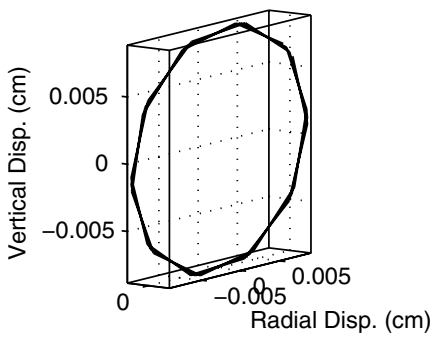

(i)

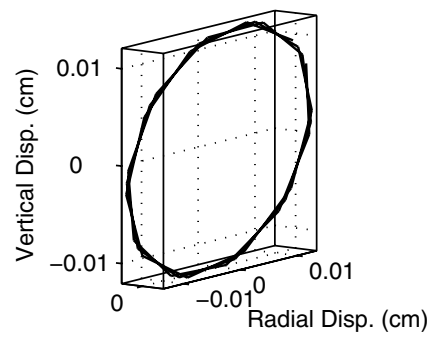

Figure 6. Sample of observed particle motion at $20 \mathrm{~Hz}$. Shown is the particle motion in the vertical-radial plane for stations 08 and 03 at the 12,000-lb applied force load (a)-(b), 36,000-lb applied force load (d)-(e), and 60,000-lb applied force load (g)-(h). Also shown is a threedimensional view of the particle motion for station 03 at the 12,000-lb applied force load (c), 36,000-lb applied force load (f), and 60,000-lb applied force load (i). See Figure 3 for the orientation of the radial and transverse directions. The retrograde particle motion is consistent with Rayleigh surface waves. Notice the lack of transverse particle motion in the three-dimensional plots.

an indicator of nonlinear ground response. Rayleigh-wave phase velocity studies are popular for estimating shallow $S$-wave velocities when assessing earthquake site response. Consequently, a number of techniques have been developed to extract the phase velocity dispersion information from ground-motion recordings (Louie, 2001). We incorporate aspects from the commonly used surface wave techniques to calculate the phase velocity dispersion in our dataset.

We first calculate the average phase velocity dispersion across the array by applying a frequency-wavenumber $(f-k)$ technique to create a slowness-frequency $(p-f)$ image. The Rayleigh phase velocity dispersion curve is picked off of the peak in the $p-f$ image. We start by filtering the vertical ground-motion data using a narrow-band-pass, 2-pole, zerophase Butterworth filter. We use 51 discrete $1-\mathrm{Hz}$ frequency bands between 5 and $30 \mathrm{~Hz}$ at every $0.5-\mathrm{Hz}$ interval (e.g., a center frequency of $15 \mathrm{~Hz}$ has a band pass of $14.5-15.5 \mathrm{~Hz}$ ). We do this for every station and for all five applied force loads. Figure $7 \mathrm{~b}$ shows an example of a step-sweep waveform band-pass filtered with a center frequency of $15 \mathrm{~Hz}$.
A time-distance $(t-x)$ plot of the filtered data clearly shows the $15-\mathrm{Hz}$ phase propagating across the array (Fig. 7c). Quantifying the velocity associated with that observed moveout for different frequency bands is the concept of the phase velocity dispersion analysis presented here. The phase moveout on $t-x$ plots for different applied force loads demonstrates how the phase velocity changes when we increase the force load. As shown in Figure 7c, a higher applied force load (and thus higher ground-motion amplitude) produces more moveout of the propagating wave across the array (i.e., lower phase velocity). This is consistent with nonlinear response because a velocity decrease is a consequence of nonlinear behavior.

To quantify the phase velocity dispersion across the array, an $f$ - $k$ transform is applied to the filtered $t-x$ data (Harris and Young, 1997). The algorithm is based on the maximumlikelihood approach (Capon, 1969). Dutta et al. (2007) show a recent example of using an $f-k$ analysis technique to extract Rayleigh phase velocity dispersion information. The $f-k$ transform is performed on all 51 frequency bands for all five 
(a)

(b)

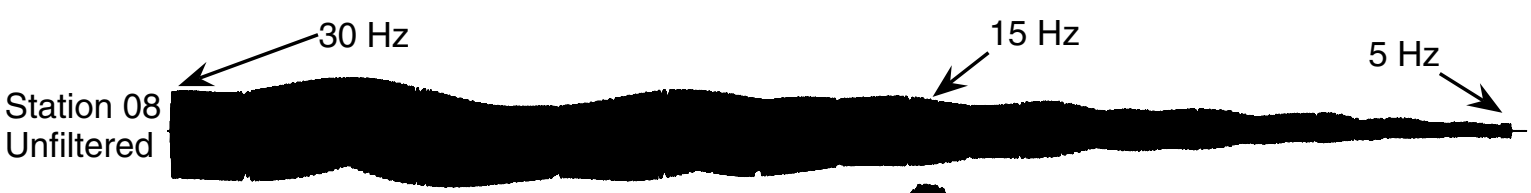

Station 08

Filtered @ $15 \mathrm{~Hz}$

(c)

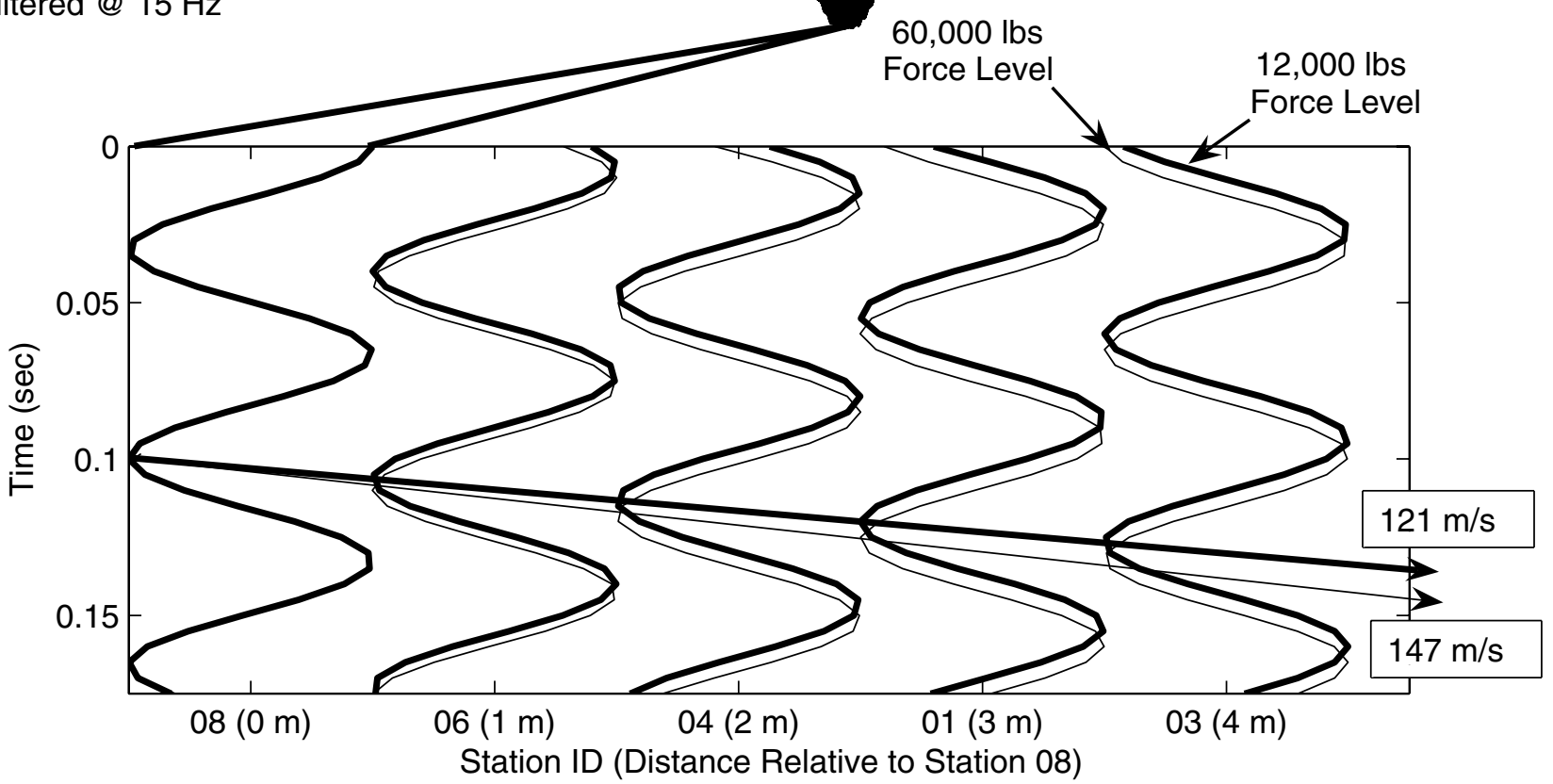

Figure 7. Example $t-x$ plot with data narrow band-pass-filtered at $15 \mathrm{~Hz}$. Unfiltered (a) and filtered (b) data for station 08 are shown. A $t-x$ plot (c) for the lowest and highest applied force loads shows the phase moveout across the array at $15 \mathrm{~Hz}$. The $t-x$ plot has been normalized to remove the effects of attenuation with distance. The lowest applied force load $(12,000 \mathrm{lb})$ has less phase moveout than the highest applied force load $(60,000 \mathrm{lb})$. This is consistent with nonlinear response occurring in the soil.

applied force loads using a range of slowness values from 0.0001 to $0.02 \mathrm{sec} / \mathrm{m}$. For each applied force load, the $f-k$ spectra of all 51 discrete frequency bands are collected to create the resulting five $p-f$ images. One of those five $p-f$ images, for the 60,000-lb force load, is seen in Figure 8. The use of the $p$ - $f$ domain to extract the phase velocity dispersion is similar to the multichannel analysis of surface waves (MASW) technique (Park et al., 1999) and the refraction microtremor (ReMi) technique (Louie, 2001) commonly employed to estimate shallow $S$-wave structure. Finally, we pick the phase velocity dispersion curve from the peak in the $p-f$ image, shown by a dotted line in Figure 8 . Uncertainty in the resulting phase velocity dispersion curves is estimated using the width of the peak in the $p-f$ image.

Figure 9 shows the resulting phase velocity dispersion curves for all five applied force loads. This is the principal result from our study. A clear trend is observed where the phase velocity decreases as applied force is increased. This is convincing evidence of induced nonlinear response in the shallow sediments because a velocity decrease is a consequence of nonlinear behavior. Between the lowest and highest applied force loads, the phase velocity values decrease as much as $\sim 30 \%$. The largest decrease in phase velocity occurs between the two lowest force loads. Between the three larg- est force loads, the phase velocity only slightly decreases. The amount of change in phase velocity between all five force loads is also a function of frequency, with more reduction at the highest frequencies. This is likely caused by the induced strain field of the surface source decreasing in am-

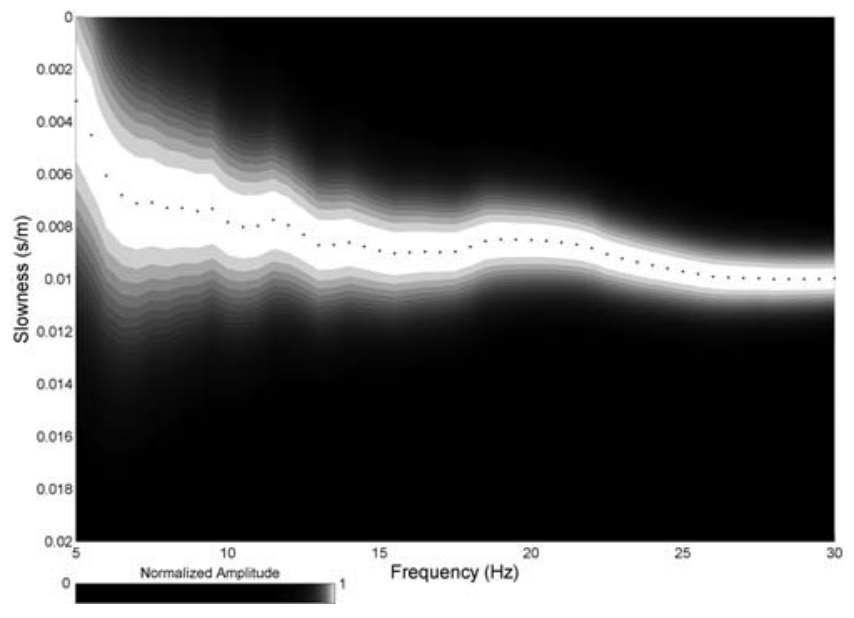

Figure 8. An example $p$ - $f$ image used to pick the Rayleigh phase velocity dispersion. The dispersion curve is shown with small dots. Shown are data for the 60,000-lb force load. 


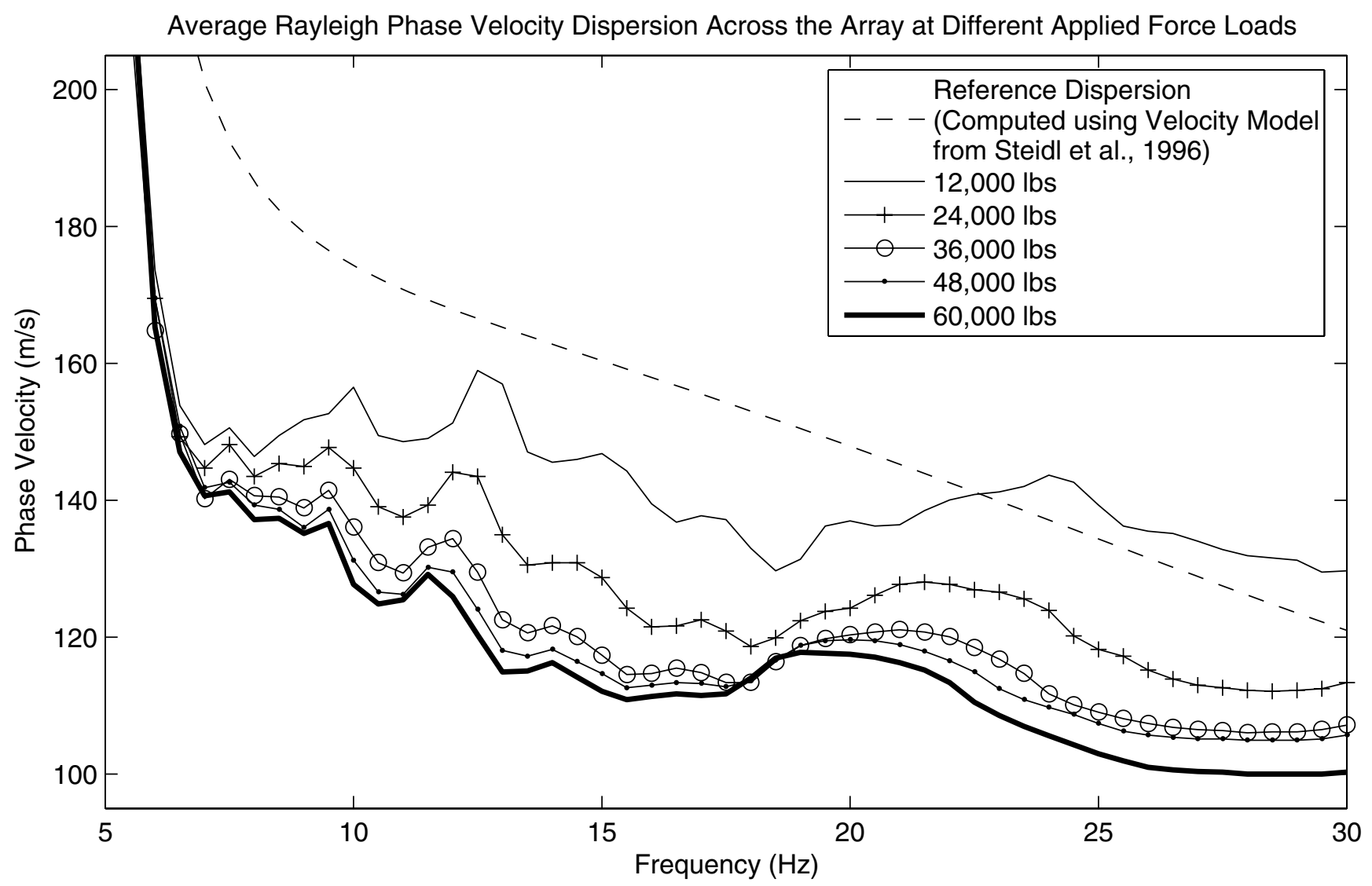

Figure 9. Average Rayleigh-wave phase velocity dispersion across the array at different applied force loads. These curves were obtained from $p$ - $f$ images similar to Figure 8. The decrease in phase velocity as the applied force load increases is due to nonlinear response in the shallow sediments. Also shown is a reference dispersion curve calculated using a Garner Valley velocity model published in Steidl et al. (1996), which we treat as the linear response dispersion curve.

plitude with depth. The higher frequency Rayleigh waves are more sensitive to the shallowest site structure compared to the long wavelengths of the lower frequency waves. Thus, more nonlinear response occurs nearest to the surface (a propagation effect). The observed reduced nonlinearity at low frequencies may also in part reflect T-Rex's decrease in force output at frequencies below $\sim 12 \mathrm{~Hz}$ (a source effect).

Figure 9 also compares our high-strain phase velocity dispersion curves to a reference small-strain dispersion curve. Steidl et al. (1996) published a velocity model for Garner Valley when modeling site response in the area. We are using this model to represent the linear response of the near-surface structure. The reference dispersion curve is slightly higher in velocity than the calculated dispersion curve for the lowest force load and much higher than the dispersion curves for the other force loads. This is consistent with the shallow sediments behaving nonlinearly from the large loads applied. However, the exact relationship between the reference dispersion curve and our calculated dispersion curves should not be taken too far. The Garner Valley velocity model of Steidl et al. (1996) is based on geotechnical testing and near-surface seismic site characterization. Not all reported $S$-wave velocities for Garner Valley are consistent for the upper few meters. At the frequency range we are using, variations of the $S$-wave velocity structure in the upper few meters will have a significant impact on the resulting phase velocity values. Steidl et al. (1996) use an $S$-wave velocity of $90 \mathrm{~m} / \mathrm{sec}$ for the uppermost 1-m-thick layer, which is based on geotechnical testing and $S$-wave velocity profiles from Pecker (1995). However, Brown et al. (2002) and Stokoe, Kurtulus, and Meng (2004) used the spectral analysis of surface waves (SASW) technique (Nazarian and Desai, 1993) to estimate the $S$-wave velocity structure (under linear conditions) at the Garner Valley test site. Results from Brown et al. (2002) did not adequately resolve the velocity structure in the upper few meters. Results from five SASW lines in Stokoe, Kurtulus, and Meng (2004) show a variation of modeled $S$-wave velocities for the upper $5 \mathrm{~m}$. For instance, the modeled $S$-wave velocity for the top layer in the five seismic lines in Stokoe, Kurtulus, and Meng (2004) ranges from 137 to $207 \mathrm{~m} / \mathrm{sec}$, higher than the $90 \mathrm{~m} / \mathrm{sec}$ velocity cited in Steidl et al. (1996) for the upper 1-m-thick layer.

The $f$ - $k$ phase velocity analysis described averages the travel time of the wave field over the whole array. This minimizes the travel time differences that might exist within the array due to station location errors or soil heterogeneity. The 
variation of reported $S$-wave velocities for Garner Valley suggests that some degree of soil heterogeneity exists. Station location errors may also be troublesome given that the size of a single $\mathrm{K} 2$ accelerometer is one-fifth of the radial station spacing. Those reasons are why we prefer the $f$ - $k$ analysis approach that averages the phase velocity over the entire array. However, there is a compelling reason to calculate the interstation phase velocity as well. If the ground-motion amplitude can decrease by two-thirds over the 4-m-long array, it seems reasonable that more nonlinearity occurs closer to T-Rex where the ground accelerations are highest.

To calculate the phase velocity dispersion between successive station pairs, we employ the standard two-station phase difference method (Aki and Richards, 1987). This is also similar to the SASW technique commonly employed to estimate shallow $S$-wave velocity structure. We use the same data as before: vertical component ground motion created by a vertically excited harmonic step sweep at five applied force loads. We obtain the phase velocity dispersion between two stations by

$$
V(\omega)=\frac{-2 \omega \Delta x}{\phi_{2}(\omega)-\phi_{1}(\omega)},
$$

where $V(\omega)$ is the phase velocity dispersion function, $\omega=2 \pi f, f$ is the discrete frequency, $\phi(\omega)$ is the Fourier phase spectrum of the vertical ground-motion time series for a particular station using the fast Fourier transform (FFT), and $\Delta x$ is the distance between the two stations. We restrict ourselves to the bandwidth of the step sweep, 5-30 Hz. We do not smooth the phase velocity spectrum resulting from equation (1). The long duration time series ( 6.5 min) results in extremely closely sampled spectral data points. The phase velocity measurements are subject to aliasing associated with the phase wavelength and station spacing. Our array has a radial station spacing of $1.0 \mathrm{~m}$ and at the highest frequency (shortest wavelength), aliasing will occur below a phase velocity of $60 \mathrm{~m} / \mathrm{sec}$.

Figure 10 shows the phase velocity dispersion results using four successive station pairs. The station pairs are, in order of increasing distance from T-Rex, 08-06, 06-04, 04-01, and 01-03 (see Fig. 3 for station locations). The station pairs span the length of the array in the radial direction relative to T-Rex and the direction of wave propagation. Because all stations equidistant from T-Rex are observed to be in phase, the station pairs used are treated as if they are oriented strictly perpendicular from T-Rex even though they are staggered slightly in the transverse direction. Each dispersion curve in Figure 10 is a finely sampled scatter plot. The width of the scatter for each dispersion curve is used to give an estimate of the uncertainty.

Figure 10 shows that phase velocity decreases as the applied force load is increased. This is consistent with results from the phase velocity dispersion curves averaged over the entire array shown in Figure 9. The shape and value of the dispersion curves have a significant amount of variance between station pairs. This is the reason why we prefer the array-averaged dispersion curves (Fig. 9). However, there are some interesting observations regarding the dispersion between individual stations. The dispersion curves for station pair 08-06 (Fig. 10a) share the general shape as the dispersion curves in Figure 9 only with lower phase velocity values. In fact, the phase velocity values generally increase for the station pairs farther from T-Rex. Furthermore, at station pairs farther from T-Rex, the decrease in phase velocity as a function of applied force load is less. Station pairs 08-06 and 06-04, which are closest to T-Rex, show the lowest phase velocity values and greatest amount of velocity decrease as force load is increased. Some frequency ranges for station pairs 04-01 and 01-03 do not show a decrease in phase velocity as the force load is increased at all. Still, the dispersion curves between successive stations (Fig. 10) confirm the observations in Figure 9 that phase velocity is observed to be a function of the applied force load. The interstation phase velocity dispersion curves also suggest that more nonlinear response is occurring closer to T-Rex where the ground-motion amplitudes are largest.

We checked the phase velocity dispersion results in Figures 9 and 10 by handpicking arrival times of troughs and peaks of phases across the array as well as with an automated approach using cross correlation. Results using these simple but time exhaustive time domain methods confirm the phase velocity values in Figures 9 and 10. We also compute phase velocity dispersion curves for the radial component of motion using all of the same methods. The resulting radialcomponent dispersion curves (not shown) are very similar to the vertical component, consistent with Rayleigh-wave propagation. We do not make any effort to remove body wave energy from the ground motions.

A particularly striking feature observed in the dispersion curves in Figures 9 and 10 is the variation with frequency, seen as a series of peaks and troughs, making the curves look rough. If the velocity structure increases monotonically with depth, the expected phase velocity dispersion curves should be smoothly decreasing with increasing frequency. Increasing phase velocity with increasing frequency is a hallmark of a velocity reversal, or low-velocity zone, in the subsurface velocity structure. The Garner Valley velocity model used by Steidl et al. (1996) does not include any low-velocity zones with depth. The available geotechnical logs (J. Steidl, personal comm., 2004) also show that there are not any observed low-velocity zones large enough to produce the amplitude of the roughness seen in our phase velocity dispersion curves. Therefore, it is possible that the features that comprise the roughness of the dispersion curves, although systematic and repeatable in our data, do not result from low-velocity zones in the near-surface velocity structure.

The small peaks and troughs present in the observed dispersion curves in Figures 9 and 10 curiously shift to lower frequency as applied force load is increased. We suggest that this could be due to the interference between fundamental and higher Rayleigh-wave modes. We test this idea by com- 
Inter-Station Phase Velocity Dispersion
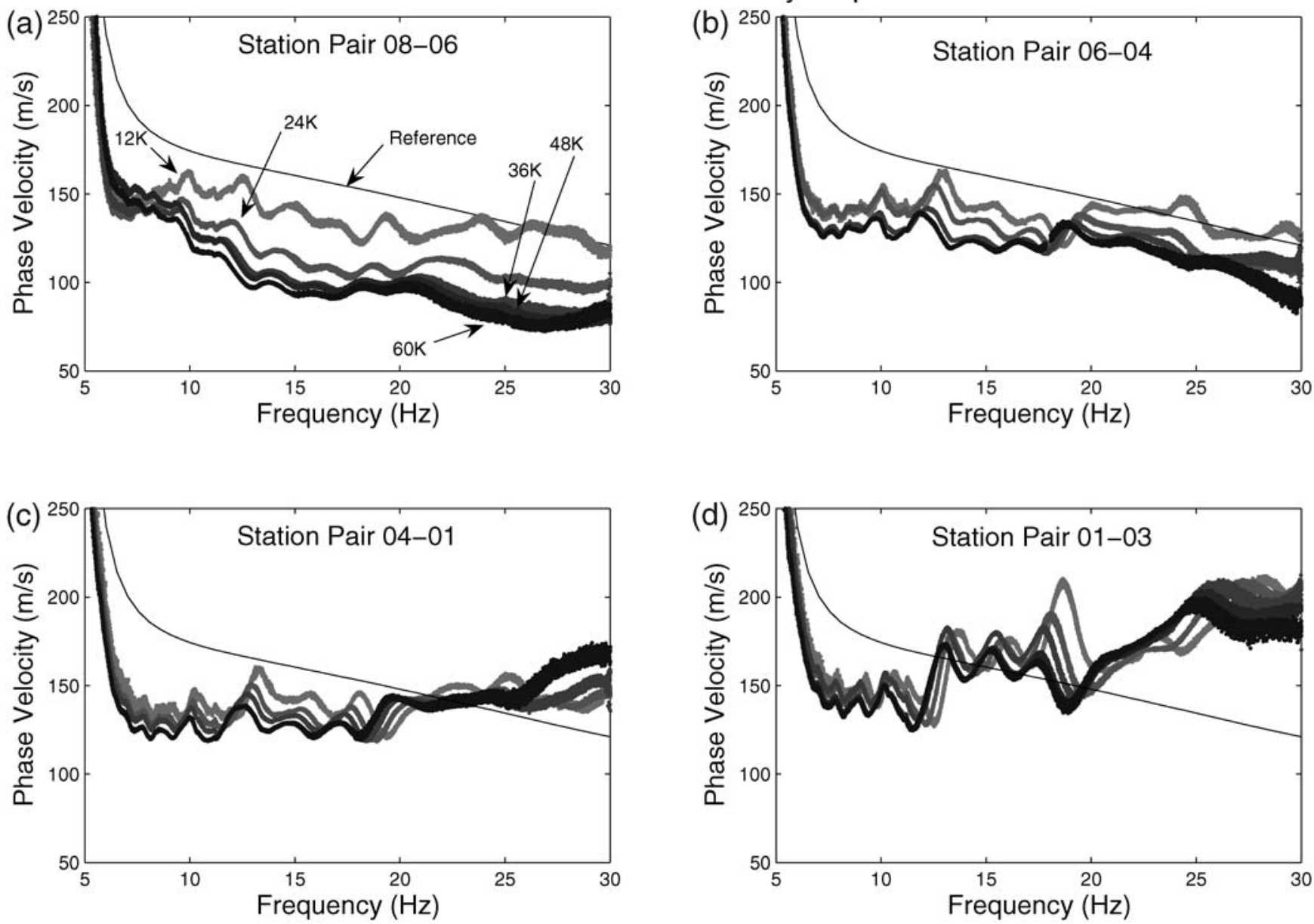

Figure 10. Interstation Rayleigh phase velocity dispersion curves at different applied force loads. Shown are station pairs 08-06 (a), 0604 (b), 04-01 (c), and 01-03 (d). These station pairs span the entire length of the array in a radial direction away from T-Rex. Each observed dispersion curve is a finely sampled scatter plot. The darker shade of gray represents a higher applied force load. The lightest shade of gray corresponds to the $12,000 \mathrm{lb}$. The darkest shade of gray corresponds to $60,000 \mathrm{lb}$. The solid black line labeled reference is the same reference dispersion curve shown in Figure 9, which was calculated using a Garner Valley velocity model published in Steidl et al. (1996). Note that for some station pairs, the dispersion curves overlap and are difficult to distinguish. Station pairs 06-04 (b) and 04-01 (c) do not have a 48,000-lb force load due to data loss during collection.

puting fundamental and higher mode group velocity dispersion curves for the Garner Valley velocity model in Steidl et al. (1996) and then again for the same model with velocities decreased by $10 \%$ to represent a nonlinear change. We use group velocity dispersion curves because when Rayleigh-wave modes interfere with one another in distance, it is their group velocity that defines the interference and not phase velocity. We find that the fundamental and secondhigher mode have the same group velocity at $\sim 13 \mathrm{~Hz}$. When the velocity structure is lowered by $10 \%$, the crossing of the fundamental and second-higher mode then occurs at $\sim 12 \mathrm{~Hz}$. This observation is fairly consistent. If a higher mode interferes with the fundamental mode, a similar yet slightly reduced velocity structure pushes the interference point to lower frequency. We proceed assuming the fundamental mode Rayleigh wave dominates the ground motions and resulting phase velocity dispersion curves. If any of the rough- ness seen in the dispersion curves is caused by interfering higher modes and not by low-velocity zones, the minor contribution fluctuates around this dominant fundamental mode signal. In the following velocity modeling, the small peaks and troughs in the dispersion curves are smoothed over in the inversion algorithm.

\section{Phase Velocity Inversion for $S$-Wave Velocity Structure}

We invert the phase velocity dispersion curves in Figures 9 and 10 for $S$-wave velocity structure assuming the wave field is dominated by fundamental mode Rayleigh waves. Inversion of Rayleigh phase velocity dispersion curves for an $S$-wave velocity structure is a nonunique problem. Many different velocity models can produce the same phase velocity dispersion curve. However, the practice of 
using the Rayleigh-wave dispersion information for estimating $S$-wave structure is an acceptable method for engineering purposes when near-surface $S$-wave structure is needed for seismic hazard calculations. The ReMi and MASW techniques have shown to match borehole $S$-wave velocities to within $15 \%$ (Stephenson et al., 2005). Rayleigh-wave dispersion is typically only used to estimate $S$-wave structure because Rayleigh waves are most sensitive to the $S$-wave structure (Lai and Rix, 1998). Small changes in $S$-wave velocity can produce a significant change in Rayleigh phase velocity, while even large changes in the $P$-wave velocity structure have little effect.

We proceed to this step after stating several caveats relevant to the Garner Valley data. First, we do not try to fit the small-scale variations in the dispersion curves, assuming that the smooth first-order trends reflect the structure controlling Rayleigh energy propagating in fundamental mode, as discussed in the previous paragraph. The inversion of Rayleigh waves also implicitly assumes a linear wave propagation model, yet we have strong evidence of wave propagation nonlinearity at the site. However, we justify the approach because the dispersion curves represent the steady-state propagation condition at any moment in the experiment, and hence are, in ensemble, an equivalent linear estimate of the true nonlinear moduli. That is, the material has softened (and damping has increased) but the physics of the propagation of a wave has not changed markedly. Another caveat involves the assumption that waves associated with all frequencies in the dispersion curves are sampling the same velocity structure (albeit with different frequencies being sensitive to different depths). That may or may not be the case for these data because the velocity structure may be changing in time throughout the source sweep, which is over 6-min long. Each wave frequency is excited at different times, and if the velocity structure becomes altered at the beginning of the sweep at high frequencies, the subsequent lower frequency waves may interact with a different velocity structure than the higher frequency waves did.

We begin by inverting the array-averaged dispersion curves in Figure 9 for an $S$-wave velocity model for each force load using an inversion algorithm by Lai and Rix (1998). The method employs an Occam's inversion scheme (Constable et al., 1987) that uses a smoothing function with a generalized jumping inversion. We only use a bandwidth of $12-30 \mathrm{~Hz}$ during the inversion of the array-averaged dispersion curves of Figure 9. The reason is twofold. First, the force response curves for T-Rex fall off below $12 \mathrm{~Hz}$ (see Figs. 2 and 5a), and we want all of the phase velocity values being inverted to be associated with the same applied force. Second, the algorithm had a difficult time converging when using frequencies lower than $12 \mathrm{~Hz}$. Discarding lower frequency data will limit the depth to which $S$-wave velocities are modeled. The modeled one-dimensional velocity structure is parameterized to match the interfaces and layer thicknesses used in Steidl et al. (1996). Phase velocity uncertainties used in the inversion are estimated from the width of the maximum peak in the $p-f$ images. We begin by inverting the dispersion curve for the lowest force level $(12,000 \mathrm{lb})$ using the Garner Valley velocity model of Steidl et al. (1996) as the starting model. We then use the resulting $S$-wave velocity model for the 12,000 -lb dispersion curve as the starting model for the inversion of the dispersion curve for the next highest force load $(24,000 \mathrm{lb})$. We also use a similarly reduced $P$-wave velocity structure by assuming the Poisson ratio implied by Steidl et al. (1996) does not change. This process is repeated for all five force loads.

Figure 11a shows the resulting $S$-wave profiles. Only the upper $4 \mathrm{~m}$ is shown to emphasize the change in $S$-wave velocity as a function of force load in the shallowest sediments. Below $4 \mathrm{~m}$, the velocity variation as a function of force load is small compared to the calculated uncertainties. The comparison of synthetic and observed dispersion curves is shown in Figure 12. Our modeled dispersion curves for each force load adequately model the first-order trends in the experimental dispersion curves while not accurately resolving the roughness. Modeling more closely the shape of the experimental dispersion curves could sometimes be accomplished by reducing the smoothing parameters in the inversion code. However, this often had the effect of causing the inversion to diverge. Figure 11d shows the upper $4 \mathrm{~m}$ in the Garner Valley reference velocity model from Steidl et al. (1996), although we are less interested in how the modeled $S$-wave velocity profiles compare to the reference model and more so how the modeled velocities change as a function of applied force load.

As one might expect, there exist many similarities between the calculated dispersion curves in Figure 9 and the modeled $S$-wave profiles in Figure 11a. In Figure 11a, as the force load is increased, the $S$-wave velocity generally decreases. This is not an exact correlation, however. In the upper 1-m layer with increasing force load, the modeled $S$-wave velocities are 127, 92, 94, 97, and $91 \mathrm{~m} / \mathrm{sec}$, respectively, although the average $S$-wave velocity over the upper $4 \mathrm{~m}$ shown in Figure 11a is 144, 126, 119, 117, and $114 \mathrm{~m} / \mathrm{sec}$, respectively. Consistent with the observation of the dispersion curves in Figure 9 is that as the force load is increased, the site's velocity decreases. In addition, the upper 1-m-thick layer shows the most velocity reduction between the lowest and highest force loads, just as the highest frequencies showed a greater velocity reduction in the dispersion curves in Figure 9. The greatest amount of reduction in the $S$-wave velocity occurs between the first and second force loads-again, consistent for what is observed in the dispersion curves in Figure 9.

Next, we invert the interstation dispersion curves in Figure 10 for an $S$-wave velocity model for each force load. This is done in a similar manner as mentioned previously. However, we had a more difficult time inverting the interstation dispersion curves compared to the array-averaged dispersion curves. We only show inversion results for station pairs 0806 and 06-04. Station pairs 04-01 and 01-03 were difficult to model due to the increase in phase velocity at high frequen- 


\section{S-wave Velocity Profiles}

\section{(a) Inverted from Array-averaged Dispersion Curves}
(b) Inverted from Station Pair 08-06 Dispersion Curves
(c) Inverted from
Station Pair 06-04 Dispersion Curves
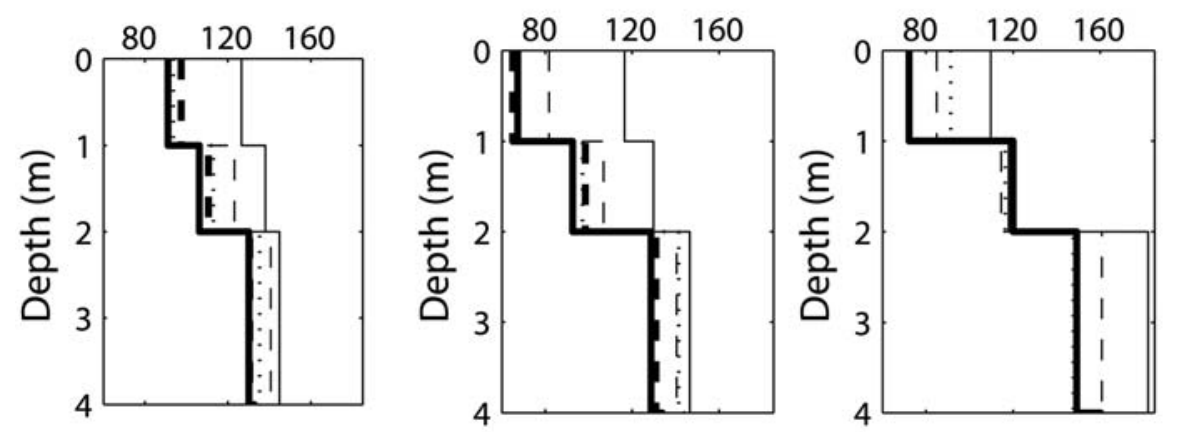

\section{(d) Reference from Steidl et al. (1996)}

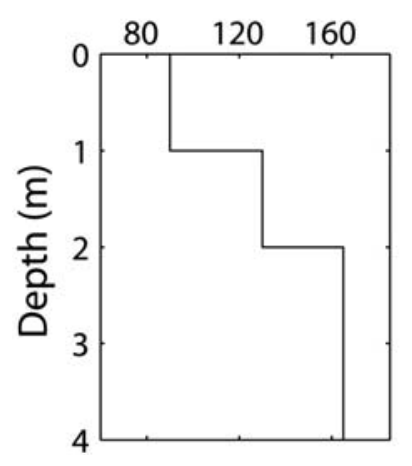

Applied Force Load (referring to $a, b, c$ )

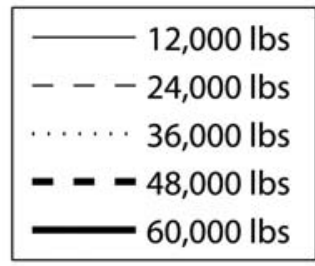

Figure 11. Estimated $S$-wave velocity profiles for the upper $4 \mathrm{~m}$ found by inverting the dispersion curves in Figures 9 and 10 . (a) The $S$-wave velocity structure inverted from the array-averaged dispersion curves in Figure 9 . (b) The $S$-wave velocity structure inverted from the interstation dispersion between station pair 08-06 in Figure 10. (c) The $S$-wave velocity structure inverted from the interstation dispersion between station pair 06-04 in Figure 10. Station pair 06-04 does not have a 48,000-lb curve due to loss of data during collection. (d) The $S$-wave velocity structure from Steidl et al. (1996) for the upper $4 \mathrm{~m}$.

cies, which caused the inversion to include numerous and sometimes large low-velocity zones in the final model, if the inversion converged at all. Results from the array-averaged dispersion curves suggest that most of the nonlinear response is occurring in the top $4 \mathrm{~m}$. Therefore, we decided to only use wavelengths sensitive to the upper $4 \mathrm{~m}$ in the inversion of the interstation dispersion curves to help with convergence. We used a half-wavelength approximation to estimate the maximum model depth needed given phase velocity at the lowest frequency data point (Park et al., 1999). Using dispersion data in Figure 10a and b between 20 and $30 \mathrm{~Hz}$ ensures that the frequencies would be sensitive to only the upper $4 \mathrm{~m}$ (i.e., at $20 \mathrm{~Hz}$, the wave will need to go faster than $160 \mathrm{~m} / \mathrm{sec}$ to be sensitive to below $4 \mathrm{~m}$ ).

Figure $11 \mathrm{~b}$ and c shows the modeled $S$-wave velocity structure for the interstation phase velocity dispersion of station pairs 08-06 and 06-04. Figure 12 shows how the modeled dispersion curves compare to the experimental dispersion curves. In general, the modeled $S$-wave velocity profiles for the interstation dispersion curves in Figure 11b and $\mathrm{c}$ are similar to those for the array-averaged dispersion curves in Figure 11a. Modeled $S$-wave velocity values between station pairs 08-06 and 06-04 are generally lower than that for the array-averaged values. Again, as the force load increases, the modeled $S$-wave velocity values decrease, with some minor exceptions for certain layers.

\section{Shear Modulus Reduction}

Nonlinear soil behavior is typically expressed in terms of shear modulus reduction and material damping as a function of shear strain. We estimated $S$-wave velocity profiles for different force loads (Fig. 11) that show a general decrease in 
velocity as force load is increased. An estimate of shear strain for each force load would allow for shear modulus reduction curves to be examined. Because Rayleigh surface waves dominate the observed ground motions, we are concerned with the shear strain component across the vertical-radial plane. If we assign the radial direction as the $x$ axis, the transverse direction as the $y$ axis, and the vertical direction as the $z$ axis, the shear strain of interest is

$$
\varepsilon_{x z}=\frac{1}{2}\left(\frac{\partial u_{z}}{\partial x}+\frac{\partial u_{x}}{\partial z}\right) .
$$

\section{Array-averaged Dispersion vs Modeled Dispersion}

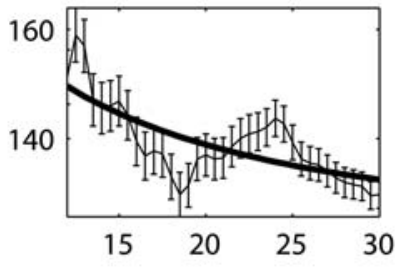

Applied Force Load

$12,000 \mathrm{lbs}$

where $u_{z 1}$ and $u_{z 2}$ are vertical displacement time series of

With sensors only on the surface, we cannot determine displacement gradients in the vertical, or $z$, direction. We expect the vertical displacement gradient $\left(\partial u_{x} / \partial z\right)$ to be of the same order of magnitude as the radial displacement gradient $\left(\partial u_{z} / \partial x\right)$. Therefore, we estimate the $\varepsilon_{x z}$ shear strain using a first-order displacement difference between stations:

$$
\varepsilon_{x z} \sim \frac{d u_{z}}{d x}=\frac{u_{z 2}-u_{z 1}}{\Delta x},
$$

\section{Inter-station Dispersion vs Modeled Dispersion}

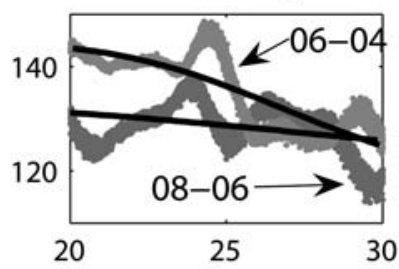

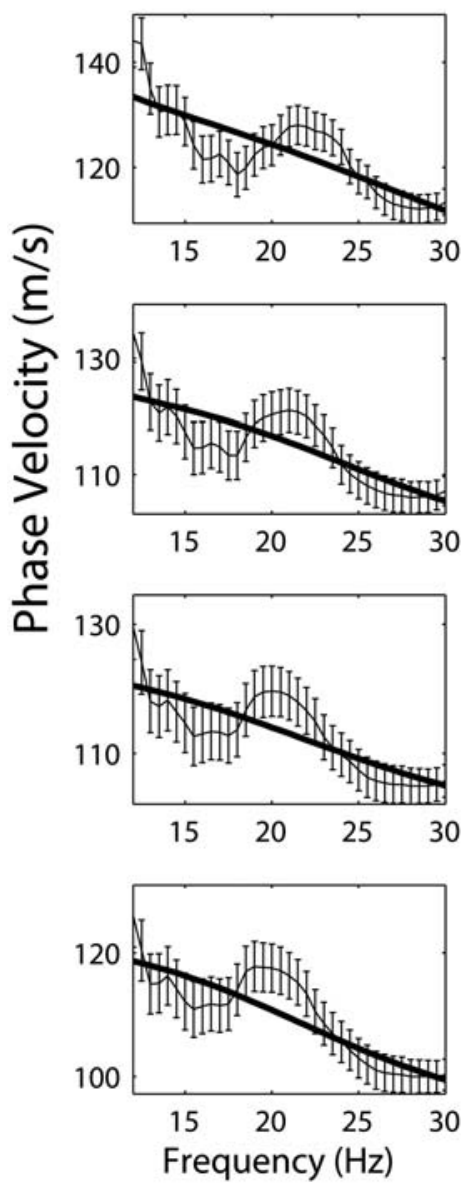
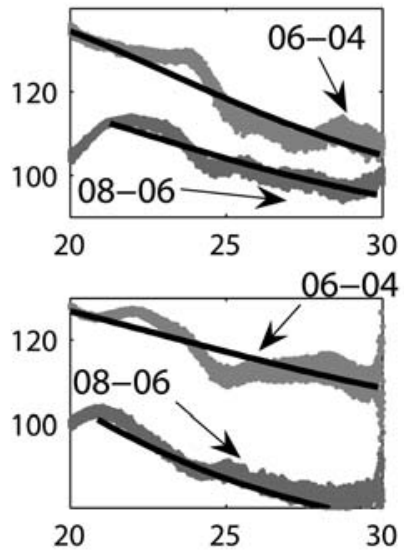

$36,000 \mathrm{lbs}$
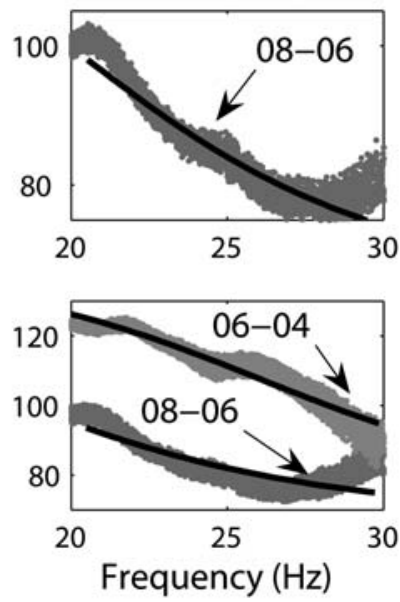

Figure 12. Observed and modeled Rayleigh-wave phase velocity dispersion curves for the array-averaged and interstation dispersion curves for all five applied force loads. Modeled dispersion curves yield good fits to the first-order trends in the dispersion data. Phase velocity uncertainties for the array-averaged dispersion curves are shown with vertical error bars. Uncertainties for the interstation dispersion curves are shown by the width of the scattered data points. Station pair 06-04 does not have data for the 48,000-lb applied force load due to data loss during collection. 
two stations and $\Delta x$ is the radial spacing between the stations. See the Appendix for our justification of this approximation. Vertical accelerations are integrated twice to obtain vertical displacement ground motions $u_{z}$. We then use the maximum value from the envelope of the strain time series along with the $S$-wave velocity values for the upper 1-mthick layer (Fig. 11) to estimate in situ shear modulus reduction values.

Shear modulus reduction curves typically normalize the shear modulus values to that of a reference low-strain, linear shear modulus (referred to as $G_{\max }$ ). What $G_{\max }$ value we use is of some concern. We believe that even at the lowest force load $(12,000 \mathrm{lb})$, some degree of nonlinear response is occurring. This is based on the large ground accelerations observed and the comparison of the 12,000-lb dispersion curve to that of the reference dispersion curve in Figure 9. However, after inverting the 12,000-lb dispersion curve for an $S$-wave profile, we found that our estimated $S$-wave value of $127 \mathrm{~m} / \mathrm{sec}$ for the upper $1 \mathrm{~m}$ is higher than the $90 \mathrm{~m} / \mathrm{sec}$ velocity in Steidl et al. (1996). However, we also know that the upper 5-10 $\mathrm{m}$ in Garner Valley likely has lateral velocity variations. This was shown by the range of velocity values modeled by Stokoe, Kurtulus, and Meng et al. (2004) using the SASW technique at the test site. Stokoe, Kurtulus, and Meng et al. (2004) modeled the upper $1 \mathrm{~m}$ to range from 137 to $207 \mathrm{~m} / \mathrm{sec}$ between their five seismic lines. We use $137 \mathrm{~m} / \mathrm{sec}$ as the linear velocity value.

Figure 13 shows the resulting in situ shear modulus reduction values plotted with a laboratory shear modulus reduction curve by Stokoe and Darendeli (1998). Given the rather large uncertainties in our in situ estimates of the shear modulus reduction, the values are of the same order as the laboratory values of Stokoe and Darendeli (1998). The laboratory curve is for a sample from 3.5-m deep at the Garner Valley test site. The uncertainties in the laboratory-derived values were not quantified. We can calculate $G / G_{\max }$ values for any layer in our velocity models. We are limited in estimating the shear strain because we only have a surface array. Thus, we only pair the shear strain estimates with $G / G_{\max }$ values for the uppermost 1 -m-thick layer. The $G / G_{\max }$ uncertainties shown in modulus reduction in Figure 13 include estimates of errors derived from computing the phase velocity dispersion curves, uncertainties associated with inversion of those dispersion curves, and uncertainties in what $G_{\max }$ is used. The uncertainties in shear strain values in Figure 13 are associated with uncertainties expected from

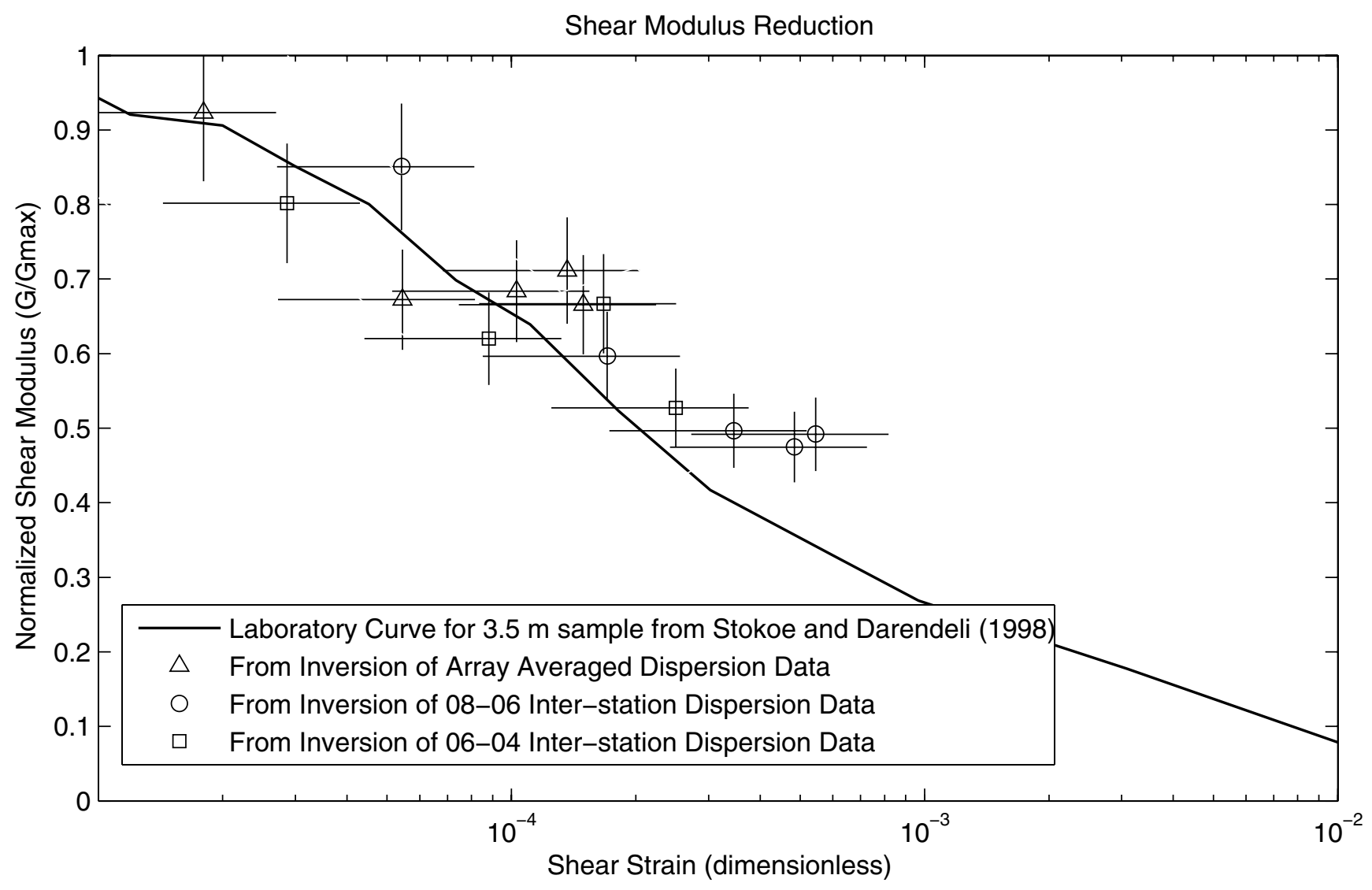

Figure 13. In situ and laboratory-derived shear modulus reduction values for the Garner Valley site. In situ values are derived from the array-averaged and interstation dispersion curves in Figures 9 and 10. $G / G_{\max }$ values were found using the estimated $S$-wave velocity values for the upper 1-m layer in Figure 11 . Velocity $137 \mathrm{~m} / \mathrm{sec}$ was used to calculate $G_{\max }$. The shear strain values were estimated using a simple finite displacement difference. Estimated uncertainties in both shear modulus and strain are shown with error bars. 
a simple first-order finite difference estimate of the spatial displacement gradient in equation (2) (Langston, 2007a). The estimated in situ shear modulus reduction values in Figure 13 contain wave propagation effects not applicable for the laboratory point measurements. Nonetheless, the comparisons of our estimated in situ shear modulus reduction values compare favorably to the laboratory-derived values and show promise that this approach to investigating in situ nonlinear response is worth future consideration for researchers trying to better understand near-surface in situ nonlinear response.

\section{Discussion}

This was a prototype experiment with the primary motivation to investigate the use of a large shaker truck to study in situ nonlinear response. While preparing and conducting the fieldwork, we were not entirely confident how we were going to use the recorded ground motions to look for or against evidence of in situ nonlinear behavior. Our initial direction was to look at the change in the fundamental resonance frequency for one of the near-surface interfaces. The fundamental resonance frequency of a layer is proportional to the wave velocity; therefore, strains large enough to cause nonlinearity will decrease the modulus values of the sediment and cause a decrease in velocity and resonant frequency (Beresnev and Wen, 1996). We found that using resonant frequency peaks to observe nonlinear sediment behavior in our data was complicated by the nonlinear nature of shaker pad interaction with the ground. We calculated site response spectra and found that resonant frequency peaks did indeed shift to a lower frequency as the force load was increased (as predicted by nonlinear response). However, we could not rule out the observations being caused by changes in the source frequency for different force loads. The interaction between the shaker pad and the ground can be a nonlinear system. That is, the ratio of applied force to the energy transmitted into the ground (as a function of frequency) may change when force load is increased. During data analysis it became clear that using amplitude changes to infer nonlinear response would be limited due to uncertainties in the T-Rex's own nonlinear behavior. The best way to avoid the source complications was to look directly at the change in velocity of the propagating wave field. Once the seismic disturbance is created in the ground, the travel time of the propagating wave should not be affected by the nonlinear behavior of the source.

Figure 9 represents the most important result from this study. Shown is the observation that as we applied larger force loads to the ground to create larger seismic strains, the measured Rayleigh phase velocity (as a function of frequency) across the array decreased. We interpret the decrease in Rayleigh phase velocity to be caused by a decrease in (primarily) shear modulus of the shallow sediments. The propagation velocity of Rayleigh surface waves is controlled primarily by the $S$-wave velocity structure. Although addi- tional theoretical work is needed to parameterize strainamplitude-dependent phase velocity in seismological inverse formulations, the simple procedure of estimating $S$-wave velocity structure by inverting the phase velocity dispersion using equivalent linear models of Rayleigh-wave propagation gave results that are sensible and empirical. The modeled $S$-wave velocity profiles and estimated shear strains permit estimated in situ shear modulus reduction values. Shear modulus reduction curves are one of the products needed in seismic hazard assessments. Our estimated in situ shear modulus reduction values compare favorably to laboratory point measurements made from the same site.

$S$-wave velocity inversion results showed that the greatest change in velocity as a function of force load occurred in the upper $4 \mathrm{~m}$. The 4-m interface is important as it represents the depth of the water table in Garner Valley during the dry summer months (Steidl et al., 1996). We are concerned with what happens to pore pressures as we shake the ground with such high ground accelerations. In our case, the modeled $S$-wave velocity values are for unsaturated sediments. Future studies using this approach will need to pay special attention to the depth of the water table and how changing pore pressures in the saturated layers affect the travel time of the propagating surface waves.

This study is a beginning step in using controlled sources to investigate in situ nonlinear behavior. Our approach has obvious limitations that likely prevent it from being considered for current seismic hazard assessment practices. The high cost of using a shaker truck may be prohibitive. The use of a source and seismic array located on the surface limits the ability to measure the nonlinear response at depth, which is required for seismic hazard calculations. Nonetheless, the ability to induce and quantify in situ nonlinear response with a noninvasive and controlled method represents a new frontier in applied soil dynamics research. At the least, this approach adds an independent observation of in situ nonlinearity occurring during the propagation highstrain seismic waves. This approach could eventually lead to new ways to quantify the nonlinear parameters of a site in situ, regardless of soil properties that may cause trouble for laboratory point measurements.

Future research using this approach needs to focus on improving the strong-motion instruments used to record the ground motions and better estimates of the seismic strain field. We used Kinemetrics K2 accelerometers, which are typical force-balance sensors. They are quite bulky in the field, thus limiting how close one can install them. Errors associated with calculating shear strains using finite displacement differences is a function of the station spacing. Smaller and lighter accelerometers, which are now available to the seismological research community, will allow arrays to be constructed with smaller station spacing. Smaller station spacing along with array-based wave gradiometry techniques (Langston, 2007a,b) will reduce the uncertainty in calculating the seismic strain field. In addition, smaller accelerometers would decrease the possibility of ground-motion 
contamination caused by the interaction between the soil and instrument pads.

\section{Conclusions}

We have successfully demonstrated that nonlinear response in near-surface sediments can be studied in situ using a large mobile shaker and nearby surface array of accelerometers. Using the NEES mobile shaker T-Rex and an array of accelerometers, we were able to produce and measure ground motions exceeding $1 g$ of ground acceleration. These large ground motions, rich in Rayleigh surface waves, induced nonlinear response in near-surface sediments. As the force load of the shaker increased, the Rayleigh-wave phase velocity (at the highest frequencies) decreased by as much as $\sim 30 \%$. Phase velocity dispersion curves were inverted for $S$-wave velocity as a function of depth. The greatest change in velocity occurred nearest the surface and T-Rex, with possibly as much as $30 \%-40 \%$ decrease in $S$-wave velocity. The estimated $S$-wave velocity values were used with shear strain estimates to calculate shear modulus reduction values that compare favorably to laboratory measurements from the same site. Overall, our experimental protocol and data analysis methods show that it is possible and efficient to drive in situ materials into their nonlinear strain regime and to measure the nonlinear response. A controlled source seismic array experiment complements laboratory studies and passive recording of earthquake data in the nascent field of material nonlinearity.

\section{Acknowledgments}

This work was supported by Mid America Earthquake Center Contract Number HD-5. The experimental portion of this work could not have been completed without the help of Jamie Steidl. Steve Horton and Robert Henke made several useful suggestions for the improvement of this manuscript. This is CERI Contribution Number 519.

\section{References}

Aguirre, J., and K. Irikura (1997). Nonlinearity, liquefaction, and velocity variation of soft soil layers in Port Island, Kobe, during the Hyogoken Nanbu earthquake, Bull. Seismol. Soc. Am. 87, $1244-1258$.

Aki, K., and P. Richards (1987). Quantitative Seismology. Theory and Methods, W. H. Freeman, San Francisco.

Beresnev, I. A., and K. L. Wen (1996). Nonlinear soil response-a reality?, Bull. Seismol. Soc. Am. 86, 1964-1978.

Bonilla, L. F., R. J. Archuleta, and D. Lavallee (2005). Hysteretic and dilatant behavior of cohesionless soils and their effects on nonlinear site response: field data observations and modeling, Bull. Seismol. Soc. Am. 95, 2373-2395.

Borcherdt, R. D. (1988). Volumetric strain and particle displacements for body and surface waves in a general viscoelastic half-space, Geophys. J. R. Astron. Soc. 93, 215-228.

Borcherdt, R. D. (2007). Characteristics of Love- and Rayleigh-type surface waves in viscolelastic media, Seism. Res. Lett. 78, 311.

Brown, L. T., D. M. Boore, and K. H. Stokoe (2002). Comparison of shearwave slowness profiles at 10 strong-motion sites from noninvasive SASW measurements and measurements made in boreholes, Bull. Seismol. Soc. Am. 92, 3116-3133.
Capon, J. (1996). High-resolution frequency-wave-number spectrum analysis, Proc. IEEE 57, 1408-1418.

Constable, S. C., R. L. Parker, and C. G. Constable (1987). Occam's inversion: a practical algorithm for generating smooth models from electromagnetic sounding data, Geophysics 52, 289-300.

Dimitriu, P. P. (1990). Preliminary results of vibrator-aided experiments in non-linear seismology concluded at Uetze, F. R. G., Phys. Earth Planet. Interiors 63, 172-180.

Dutta, U., T. Satoh, H. Kawase, T. Sato, A. Martirosyan, and M. Dravinski (2007). $S$-wave velocity structure of sediments in Anchorage, Alaska, estimated with array measurements of microtremors, Bull. Seismol. Soc. Am. 97, 234-255.

Field, E. H., P. A. Johnson, I. Beresnev, and Y. Zeng (1997). Nonlinear ground-motion amplification by sediments during the 1994 Northridge earthquake, Nature 390, 599-602.

Field, E. H., S. Kramer, A. W. Elgamal, J. D. Bray, N. Matasovic, P. A. Johnson, C. Cramer, C. Roblee, D. J. Wald, L. F. Bonilla, P. P. Dimitriu, and J. G. Anderson (1998). Nonlinear site response: where we're at (a report from a SCEC/PEER seminar and workshop), Seism. Res. Lett. 69, 230-234.

Frankel, A. D., D. L. Carver, and R. A. Williams (2002). Nonlinear and linear site response and basin effects in Seattle for M 6.8 Nisqually, Washington, earthquake, Bull. Seismol. Soc. Am. 92, 2090-2109.

Gomberg, J., and Participants in the IRIS/NEES/USGS Workshop and Garner Valley Experiment (2004). Multifacility, multidisciplinary earthquake science and engineering, EOS 85, 404.

Guyer, R. A., and P. A. Johnson (1999). The astonishing case of mesoscopic elastic nonlinearity, Phys. Today 52, 30-35.

Hardin, B. O., and V. P. Drnevich (1972a). Shear modulus and damping in soil: measurement and parameter effects, J. Soil Mech. Found. Div. ASCE 98, 603-624.

Hardin, B. O., and V. P. Drnevich (1972b). Shear modulus and damping in soil: design equations and curves, J. Soil Mech. Found. Div. ASCE 98, 667-692.

Harris, J. M., and C. J. Young (1997). MatSeis: a seismic graphical user interface and toolbox for MATLAB, Seism. Res. Lett. 68, 307-308.

Kramer, S. L. (1996). Geotechnical Earthquake Engineering, Prentice Hall, New York.

Lai, C. G., and G. J. Rix (1998). Simultaneous inversion of Rayleigh phase velocity and attenuation for near-surface site characterization, a research report funded by the National Science Foundation and the U.S. Geological Survey, Georgia Institute of Technology.

Langston, C. A. (2007a). Spatial gradient analysis for linear seismic arrays, Bull. Seismol. Soc. Am. 97, 265-280.

Langston, C. A. (2007b). Wave gradiometry in the time domain, Bull. Seismol. Soc. Am. 97, 926-933.

Louie, J. N. (2001). Faster, better: shear-wave velocity to 100 meters depth from refraction microtremor arrays, Bull. Seismol. Soc. Am. 91, 347-364.

Nazarian, S., and M. R. Desai (1993). Automated surface wave method: field testing, J. Geotech. Eng., 119, 1094-1111.

Niemunis, A. (1995). On the estimation of the amplitude of shear strain from measurements in situ, Soil Dyn. Earthquake Eng. 14, 1-3.

Ostrovsky, L., and P. A. Johnson (2001). Dynamic nonlinear elasticity in geomaterials, Riv. Nuovo Cimento 24, 1-46.

Park, C. B., R. D. Miller, and J. Xia (1999). Multichannel analysis of surface waves, Geophysics 64, 800-808.

Pecker, A. (1995). Validation of small strain properties from recorded weak seismic motions, Soil Dyn. Earthquake Eng. 14, 399-408.

Rubinstein, J. L., and G. C. Beroza (2004). Evidence for widespread nonlinear strong ground motion in the $M_{\mathrm{w}} 6.9$ Loma Prieta earthquake. Bull. Seismol. Soc. Am. 94, 1595-1608.

Seed, H. B., and I. M. Idriss (1970). Report No. EERC 70-10, Soil moduli and damping factors for dynamic response analysis, Earthquake Engineering Research Center, University of California, Berkeley. 
Steidl, J. H., A. G. Tumarkin, and R. J. Archuleta (1996). What is a reference site?, Bull. Seismol. Soc. Am. 86, 1733-1748.

Stephenson, W. J., J. N. Louie, S. Pullammanappallil, R. A. Williams, and J. K. Odum (2005). Blind shear-wave velocity comparison of ReMi and MASW results with boreholes to $200 \mathrm{~m}$ in Santa Clara Valley: implications for earthquake ground-motion assessment, Bull. Seismol. Soc. Am. 95, 2506-2516.

Stokoe, K. H., and M. B. Darendeli (1998). Laboratory evaluation of the dynamic properties of intact soil specimens, Garner Valley, California, Institute for Crustal Studies (Internal Report), 32 pp.

Stokoe, K. H., A. Kurtulus, and F. Y. Menq (2004). Data report: SASW measurements at the NEES Garner Valley Test Site, California, University of Texas, Austin (Internal Report), 1-12.

Stokoe, K. H., E. M. Rathje, C. R. Wilson, B. L. Rosenblad, and F. Y. Menq (2004). Development of the NEES large-scale mobile shakers and associated instrumentation for in situ evaluation of nonlinear characteristics and liquefaction resistance of soils, 13th World Conference on Earthquake Engineering, Vancouver, B.C. Canada, August 1-6, Paper No. 535, 10 pp.

Vucetic, M. (1994). Cyclic threshold shear strains in soil J. Geotech. Eng. 120, 2208-2228.

\section{Appendix}

Here we demonstrate why the vertical and radial displacement gradients of a Rayleigh wave are of the same order of magnitude, allowing for the shear strain to be approximated using only one of the quantities. Following Niemunis (1995), the displacement in the radial $(x)$ and vertical $(z)$ directions due to a plane Rayleigh wave can be expressed as

$$
\begin{aligned}
u_{x}(x, z, t)= & \left(-e^{-q z}+\frac{2 q s}{s^{2}+k^{2}} e^{-s z}\right) A \sin \left(\omega\left[t-\frac{x}{c}\right]\right), \\
u_{z}(x, z, t)= & -\frac{q}{k}\left(-e^{-q z}+\frac{2 k^{2}}{s^{2}+k^{2}} e^{-s z}\right) A \sin \\
& \times\left(\omega\left[t-\frac{x}{c}\right]\right),
\end{aligned}
$$

where $c$ is the Rayleigh-wave velocity, $c_{s}$ is the $S$-wave velocity, $c_{p}$ is the $P$-wave velocity, $\omega=2 \pi f, k=\frac{\omega}{c}$, $q=\sqrt{k^{2}-\left(\omega^{2} / c_{p}^{2}\right)}$, and $s=\sqrt{k^{2}-\left(\omega^{2} / c_{s}^{2}\right)}$. The shear strain component we wish to estimate is

$$
\varepsilon_{x z}=\frac{1}{2}\left(\frac{\partial u_{z}}{\partial x}+\frac{\partial u_{x}}{\partial z}\right)
$$

Differentiating equation (A3) and ignoring higher-order terms (i.e., infinitesimal strain theory) yields

$$
\begin{aligned}
\varepsilon_{x z}= & \frac{1}{2}\left(\frac{q}{k}\left[e^{-q z}-\frac{2 k^{2}}{s^{2}+k^{2}} e^{-s z}\right] \frac{\omega}{c}\right. \\
& \left.+\left[q e^{-q z}-s \frac{2 q s}{s^{2}+k^{2}} e^{-s z}\right]\right) A \sin \left(\omega\left[t-\frac{x}{c}\right]\right) .
\end{aligned}
$$

The two quantities added together in the first pair of brackets are proportional to $\partial u_{z} / \partial x$ and $\partial u_{x} / \partial z$, respectively. Using realistic velocity values related to our experiment shows that those two quantities are of the same order of magnitude, allowing us to use the approximation

$$
\varepsilon_{x z} \sim \frac{d u_{z}}{d x} .
$$

Center for Earthquake Research and Information

University of Memphis

3876 Central Ave, Suite 1

Memphis, Tennessee 38152-3050

(Z.L., C.A.L.)

Pacific Northwest Seismic Network Department of Earth Sciences University of Washington

Box 351310

Seattle, Washington 98195-7010

(P.B.)

Earth Resources Laboratory

Massachusetts Institute of Technology

Boston, Massachusetts 02139

(F.P.)

U.S. Geological Survey

University of Washington

Seattle, Washington 98195-1310

(J.G.)

Geophysics Group

Los Alamos National Laboratory

Mail Stop D443

Los Alamos, New Mexico 87545

(P.A.J.)

Department of Civil Engineering

University of Texas at Austin Geotechnical Center

301 E. Dean Keeton ECJ 9.227

Austin, Texas 78712

(F.-Y.M.)

Center for Integrative Natural Science and Mathematics

Northern Kentucky University

Highland Heights, Kentucky 41099

(T.B.) 